\title{
The mountainous Cretan dietary patterns and their relationship with cardiovascular risk factors: the Hellenic Isolated Cohorts MANOLIS study
}

\author{
Aliki-Eleni Farmaki ${ }^{1, *}$, Nigel William Rayner ${ }^{2,3,4}$, Angela Matchan ${ }^{2}$, \\ Panagiota Spiliopoulou ${ }^{1}$, Arthur Gilly ${ }^{2}$, Vasiliki Kariakli ${ }^{1}$, Chrysoula Kiagiadaki $^{5}$, \\ Emmanouil Tsafantakis ${ }^{5}$, Eleftheria Zeggini ${ }^{2}{ }^{\dagger}$ and George Dedoussis ${ }^{1}{ }^{1} \dagger$ \\ ${ }^{1}$ Department of Nutrition and Dietetics, School of Health Science and Education, Harokopio University, $70 \mathrm{El}$ \\ Venizelou Avenue, 17671 Athens, Greece: ${ }^{2}$ Wellcome Trust Sanger Institute, Cambridge, UK: ${ }^{3}$ Wellcome Trust \\ Centre for Human Genetics, University of Oxford, Oxford, UK: ${ }^{4}$ Oxford Centre for Diabetes, Endocrinology and \\ Metabolism, Churchill Hospital, Headington, UK: ${ }^{5}$ Anogia Medical Centre, Anogia, Crete, Greece
}

Submitted 10 March 2016: Final revision received 28 October 2016: Accepted 2 November 2016: First published online 19 December 2016

\begin{abstract}
Objective: We carried out de novo recruitment of a population-based cohort (MANOLIS study) and describe the specific population, which displays interesting characteristics in terms of diet and health in old age, through deep phenotyping. Design: Cross-sectional study where anthropometric, biochemical and clinical measurements were taken in addition to interview-based completion of an extensive questionnaire on health and lifestyle parameters. Dietary patterns were derived through principal component analysis based on a validated FFQ.

Setting: Geographically isolated Mylopotamos villages on Mount Idi, Crete, Greece. Subjects: Adults ( $n$ 1553).

Results: Mean age of the participants was 61.6 years and $55.8 \%$ were women. Of the population, $82.7 \%$ were overweight or obese with a significantly different prevalence between overweight men and women $(43.4 v .34 .7 \%, P=0.002)$. The majority $(70 \cdot 6 \%)$ of participants were married, while a larger proportion of women were widowed than men $(27 \cdot 8 v .3 .5 \%, P<0 \cdot 001)$. Smoking was more prevalent in men $(38.7 v .8 .2 \%, P<0.001)$, as $88.8 \%$ of women had never smoked. Four dietary patterns emerged as characteristic of the population; these were termed 'local', 'high fat and sugar, 'Greek café/tavern' and 'olive oil, fruits and vegetables'. Individuals more adherent to the local dietary pattern presented higher blood glucose $(\beta=4.026, P<0 \cdot 001)$. Similarly, individuals with higher compliance with the Greek café/tavern pattern had higher waist-to-hip ratio $(\beta=0 \cdot 012, P<0 \cdot 001)$, blood pressure $(\beta=1 \cdot 015, P=0.005)$ and cholesterol $(\beta=5.398, P<0 \cdot 001)$.

Conclusions: Profiling of the MANOLIS elderly population identifies unique unhealthy dietary patterns that are associated with cardiometabolic indices.
\end{abstract}

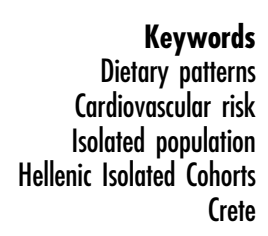

CVD are the number one cause of death globally, representing 31\% of all deaths. By 2030, almost 23.6 million people are predicted to die from CVD annually ${ }^{(1)}$. CVD is reaching epidemic proportions consistent with the worldwide sharp increase in diabetes and obesity ${ }^{(2)}$. Obesity relates to the total fat mass of an individual and is usually approximated by surrogate measurements such as BMI, waist circumference or waist-to-hip ratio (WHR). The latter two measures are more correlated to visceral fat content (central adiposity) than is BMI and are considered strong risk factors for diabetes and cardiometabolic risk ${ }^{(3,4)}$.

$\dagger$ These authors contributed equally to this work.
Risk factors associated with CVD include age, sex, ethnicity, family history, unhealthy diet, physical inactivity, smoking, alcohol consumption, obesity, hypertension, dyslipidaemia, impaired glucose and insulin metabolism, pro-thrombotic and pro-inflammatory state, and psychological and socio-economic factors ${ }^{(5,6)}$. Interestingly, most of these are associated with lifestyle and can be modified. A recent meta-analysis provided strong evidence that long-term regular lifestyle modification significantly improves many of the intermediate CVD risk factors ${ }^{(7)}$. In addition to environmental effects, a strong genetic component has also been shown to contribute to cardiometabolic disease risk $^{(8,9)}$. 
The Mediterranean diet represents a combination of many different foods in various consumption frequencies, combined with high levels of physical activity and moderate alcohol consumption, which suggests a more holistic approach to diet and lifestyle that can be incorporated in the term 'pattern'. The 'dietary pattern' approach overcomes both the conceptual and methodological limitations of the 'single nutrients' approach, taking into account the antagonistic, interactive or synergistic relationships between nutrients, as people eat meals consisting of a variety of foods and not isolated nutrients ${ }^{(10)}$.

The Seven Countries Study was the first major study to investigate diet and lifestyle along with other risk factors for $\mathrm{CVD}^{(11)}$. One of the regions studied within Greece was Crete, and the study included male residents of rural villages located inland from the northern coast, east of the capital of Heraklion. Cretan farmers were known for their longevity and held the title of the 'gold standard' of health status globally, with a very low prevalence of both CVD and cancer.

The inhabitants of mountainous Mylopotamos villages on Mount Idi, Crete, live in a geographically isolated high-altitude area and have preserved their customs, traditions and (generally) their anthropological type over the centuries. Anecdotally, this population is known to enjoy good health in old age despite a diet high in animal fat. In the present study, we established a wellphenotyped, population-based cohort in order to formally characterize the dietary and lifestyle patterns of this population and link those to traits of cardiometabolic relevance. In particular, we assessed the prevalence of diabetes and cardiovascular risk factors such as hypertension, hyperlipidaemia and obesity in this isolated population, analysed its particular dietary habits, and investigated the interactions among clinical, dietary and biochemical factors that may influence health.

\section{Methods}

\section{Study population}

The Minoan Isolates (MANOLIS) target population was adults originating from the mountainous region of Mylopotamos, on Mount Idi in Crete, which includes Anogia Municipality (2379 inhabitants), Zoniana Municipal Area (1117 inhabitants) and Livadia Local Community (1739 inhabitants). We contacted the local health centre of Anogia and with the cooperation of doctors and nurses from the regional clinics in each village, a total of 1553 volunteers were recruited between June 2010 and November 2012 (Fig. 1). According to the 2011 census, $29.7 \%$ of the total population of the region participated in the MANOLIS study.

In regional medical centres, blood samples were collected from the antecubital vein by trained health professionals following blood pressure measurement.

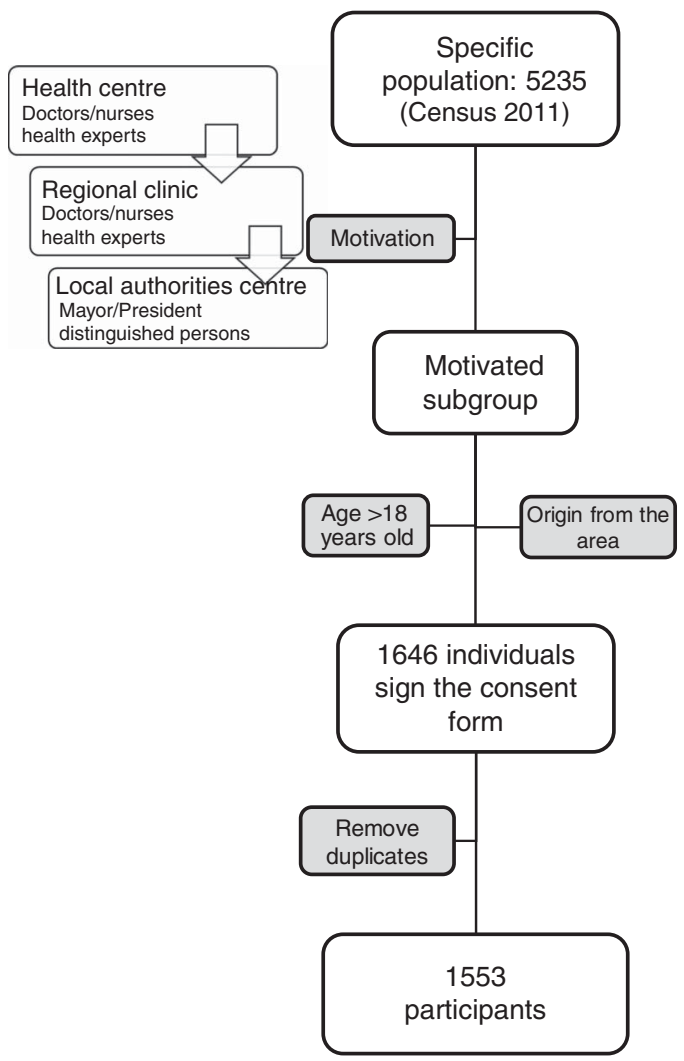

Fig. 1 Recruitment flowchart

All blood draws were conducted in the morning after an overnight fast and compliance was high.

Serum and plasma were isolated following centrifugation at $3000 \mathrm{rpm}$ for $10 \mathrm{~min}$ and stored at $-80^{\circ} \mathrm{C}$ for future analyses. All participants were interviewed by trained personnel using a standard questionnaire. The questionnaire included clinical assessment and anthropometric measurement sections, as well as questions about demographic and lifestyle characteristics, detailed medical history, physical activity and dietary habits. The medical history and corresponding medication were cross-checked and confirmed by trained clinicians.

\section{Antbropometric and biochemical measurements}

The anthropometric measurements, including weight, height, waist and hip circumferences, were conducted by trained dietitians using standardized techniques and equipment. Body weight was measured using calibrated analogue scales. For height and other anthropometric measurements a stable tape was used.

BMI was calculated as weight divided by height ${ }^{2}$ $\left(\mathrm{kg} / \mathrm{m}^{2}\right)$. BMI was used to classify participants as underweight $\left(\mathrm{BMI}<18.5 \mathrm{~kg} / \mathrm{m}^{2}\right)$, normal weight $(18.5 \leq \mathrm{BMI}$ $\left.\leq 24.9 \mathrm{~kg} / \mathrm{m}^{2}\right)$, overweight $\left(25.0 \leq \mathrm{BMI} \leq 29.9 \mathrm{~kg} / \mathrm{m}^{2}\right)$ or obese $\left(\mathrm{BMI} \geq 30 \cdot 0 \mathrm{~kg} / \mathrm{m}^{2}\right)$. WHR was also calculated.

Biochemical factors were assessed using enzymatic colorimetric assays and included glucose (hexokinase 
method), total cholesterol (cholesterol oxidase-phenol aminophenazone method), HDL cholesterol (HDL-C) and TAG (glycerol-3-phosphate oxidase-phenol aminophenazone method). Insulin was measured via chemiluminescence and LDL cholesterol (LDL-C) levels were calculated according to the Friedewald equation ${ }^{(12)}$.

\section{Demographic and lifestyle information}

Information about the marital status (married, separated/ divorced, single or widowed) of the participants was collected. Their educational level was defined as no education, primary, secondary or tertiary education, and the total number of years of education was recorded. Smoking habits were also collected. Participants were classified as current smokers, ex-smokers or never smoked. Pack-years was used as a unit for measuring the amount a person had smoked over a long period of time and was calculated by multiplying the number of packs of cigarettes (twenty cigarettes per pack) smoked per day by the number of years the person had smoked.

\section{Physical activity assessment}

Physical activity was evaluated using a brief self-reported validated questionnaire: the Harokopio Physical Activity Questionnaire (HAPAQ) ${ }^{(13)}$. The questionnaire is based on the metabolic equivalents of all activities of the preceding week, including activities at work, leisure time and rest or sleep, thus allowing the prediction of mean physical activity level and mean daily energy expenditure. The compendium of physical activities was used to quantify the intensity and consequently to calculate energy expenditure from the activities identified in the questionnaire using the method of metabolic equivalents ${ }^{(14)}$.

Based on the HAPAQ and its corresponding tool for estimating the necessary variables from the data obtained through the questionnaire, a script, the Physical Activity Program, was developed for time and memory saving purposes in order to derive these estimations directly and save them in one file (http://www.well.ox.ac.uk/ $\sim$ wrayner/tools/). This script was written in Perl and can be globally used to transform physical activity data into physical activity level, total energy expenditure and any other intermediate calculations desired.

\section{Dietary assessment}

Dietary information was collected via a validated, semi-quantitative, seventy-six-item $\mathrm{FFQ}^{(15)}$. All participants reported their daily, weekly or monthly average intake of several foods during the last year. Then the frequency of consumption was quantified on the basis of servings per week according to the dietary guidelines for adults in Greece $^{(16)}$. Alcohol consumption was also assessed and calculated as drinks per day.

The above-mentioned transformations (foods and food groups in servings) were calculated programmatically.
Energy intake (EI) was extracted from the FFQ. The sex, weight and age data of participants were used to estimate their BMR with the Schofield equations and EI:BMR was calculated in order to identify and exclude energy underreporters with the Goldberg et al. $\operatorname{method}^{(17,18)}$. Thus, a physiologically plausible EI:BMR value for our study is 1.15. Individuals whose EI:BMR was below this cut-off point were defined as under-reporters and excluded from subsequent dietary analyses ( $\left.n_{\text {final }} 1183\right)$.

\section{Statistical analysis}

Principal component analysis (PCA) was conducted to identify underlying dietary patterns ${ }^{(19)}$. In order for PCA to be effective in assessing food patterns, strong correlations between the predictive variables should exist. The correlation matrix of the food variables used in the present analysis showed that there were several correlation coefficients $(r)>|0.4|$, indicating that the variables were highly correlated. Moreover, the Kaiser-Meyer-Olkin test of sphericity and Barlett's criterion was 0.76 , implying high interrelationships between food variables and suitability of the data set for PCA. The orthogonal rotation (varimax option) was used to derive optimal non-correlated components (dietary patterns). From the entire database fifteen foods or food groups were used. To decide the number of components to retain the Kaiser criterion was used, according to which the number of components that should be retained from PCA is equal to the number of eigenvalues that are greater than 1. In our analysis, a four-component (food pattern) solution was selected. Based on the fact that factor loadings/correlation coefficients represent the correlation of each predicting variable with the dietary pattern score, higher absolute values indicate that the variable contributes more to the construction of this particular pattern. The dietary patterns were named according to scores of the predicting variables that correlated most with the component/pattern $(>|0.4|)$. The median was used to divide pattern scoring into two groups: low and high. The PCA was performed using the statistical software package IBM SPSS Statistics for Windows, Version 21.0. The data handling, basic processing and descriptive characteristics analysis were carried out in R. Outliers were defined as values that exceed 3 SD above or below the mean and were removed from subsequent analyses.

Hypothesis testing was performed using the $\chi^{2}$ test for categorical variables and Student's $t$ test or the MannWhitney for continuous variables in two different groups.

Multiple linear regression modelling was used to examine the associations between cardiometabolic indices and dietary patterns. In particular, three different models were applied: Model 1 was unadjusted; Model 2 included adjustment for age, sex, BMI (with the exception of BMI and WHR, in which analyses BMI was not a covariate), corresponding medication (for hypertension, hyperlipidaemia and diabetes), smoking in pack-years, physical 
activity level and EI; and Model 3, as per Model 2 with additional adjustment for the rest of the dietary patterns as these patterns are statistically independent, so it is possible for an individual to have high or low scores on more than one pattern at the same time and therefore all the patterns might act in concert ${ }^{(20)}$. The results from the linear regression models are presented as beta coefficients $(\beta)$ and $95 \%$ confidence intervals. The linear regressions were performed in IBM SPSS Statistics for Windows, Version 21.0. In all statistical analyses, the level of nominal significance was set at $P=0 \cdot 05$.

\section{Results}

The anthropometric and clinical characteristics of the population are presented in Table 1 . In total, 1553 individuals were recruited ( $55.8 \%$ women and $44.2 \%$ men) with a mean age of 61.6 years. Men had higher waist circumference $(103.6 v .97 .0 \mathrm{~cm})$ and WHR (0.98 v. 0.89) compared with women. The mean value for systolic blood pressure (SBP) was $138 \mathrm{mmHg}$, with men presenting significantly a higher level than women (140.1 $v$. $136.7 \mathrm{mmHg}$ ). Only $17.3 \%$ of the participants were categorized as normal weight while the vast majority ( $82.7 \%)$ were categorized as overweight or obese. Vegetables, refined cereals, fruits, meat and dairy were the food groups of the highest weekly consumption. Obesity, hypertension and hyperlipidaemia were the most prevalent diseases in this population (Table 2), with women showing higher morbidity than men.

The sociodemographic and lifestyle characteristics of the population are presented in Table 3. The majority (70.6\%) of participants were married, while a larger proportion of the women were widowed (27.8\%) compared with the men $(3.5 \%)$. The majority of participants reported primary education (63.0\%) and 10.6\% were illiterate. Men had significantly more total years of education than women ( $6.7 v .5 .5$ years). A sex-specific difference was found for both current and former smokers, with the proportion of men being four and ten times higher, respectively, than that of women. Moreover, men were found to smoke for significantly longer, presented earlier starting and later quitting age points, and smoked more packs per year than women. Sixty per cent of the population had never smoked, the vast majority of whom were women $(81 \cdot 6 \%)$.

From the initial fifty foods and food groups, fifteen were included in the PCA because of their high intercorrelation level. The Kaiser-Meyer-Olkin criterion was equal to 0.76 and the four different diet components explained $51.5 \%$ of the total variance of the examined variables. Given that higher absolute values of the score coefficients derived from PCA (see online supplementary material, Supplemental Table 1) indicate that the food contributes more to the development of the component, the components were defined as follows: (i) the 'local' diet (component 1). which includes homemade pies, legumes, red meat, full-fat cheese and refined cereals; (ii) 'high fat and sugar' diet (component 2) that consists of chocolate, milky and starchy sweets, simple sugars, ready-made pies and cheese-flavoured snacks/chips; (iii) the 'Greek café/ tavern' pattern (component 3) that is characterized by coffee and alcohol consumption, olives as a side for alcohol and fries; and (iv) the olive oil, fruits and vegetables' pattern (component 4).

The data from biochemical analyses showed that women had significantly higher mean serum levels of HDL-C than men, while men had significantly higher mean glucose, LDL-C and TAG levels compared with women. Notably, mean total cholesterol levels were above the normal range $(>200 \mathrm{mg} / \mathrm{dl})^{(21)}$ for this population (Table 4). The median was used to divide pattern scoring into two groups: low and high. The group with higher scores of the local pattern had higher mean glucose, while the groups with higher scores of the remaining patterns had lower glucose levels. Mean HDL-C was lower in the group having high scores of the Greek café/tavern pattern, while LDL-C and total cholesterol levels were found to be higher in the group with high scores of both the high fat and sugar and Greek café/tavern patterns. The group with higher scoring on the Greek café/tavern pattern presented higher TAG levels compared with the group with lower scoring on the same pattern and the group with higher scoring on the oil, fruits and vegetables pattern had higher insulin levels.

The results of the multi-adjusted linear regression analysis (Table 5) showed that component 1 , representing the local typical diet, was initially associated with BMI, WHR, SBP and diastolic blood pressure (DBP), but the associations were attenuated after adjusting for confounding factors. The local typical diet was found to be consistently associated with serum glucose levels $(\beta=4 \cdot 861, P<0 \cdot 001$; $\beta=4.618, \quad P<0.001$ after adjusting for confounders; $\beta=4.026, P<0.001$ after adjusting for confounders and the rest of the dietary patterns). Component 2 with high fat and sugar content was found to be negatively associated with BMI, WHR, HDL-C and SBP, but all these associations became non-significant after adjusting for confounding factors. In addition, the high fat and sugar dietary pattern was found to be associated with reduced glucose levels even after adjustment for confounders but not when the remaining diet was taken into account.

Component 3, representing the Greek café/tavern pattern, was associated with reduced HDL-C but the effect was non-significant after adjusting for confounding factors. A consistent association of the Greek café/tavern pattern was found with increased WHR $(\beta=0.020, P<0.001$; $\beta=0.012, \quad P<0.001$ after adjusting for confounders; $\beta=0.012, P<0.001$ after adjusting for confounders and the rest of the dietary patterns), DBP $(\beta=0.903, P=0.002$; $\beta=0.846, \quad P=0.014$ after adjusting for confounders; 
Table 1 Anthropometric, clinical and dietary characteristics of the study population by sex: adults from isolated Mylopotamos villages, Crete, Greece, the Hellenic Isolated Cohorts MANOLIS study

\begin{tabular}{|c|c|c|c|c|c|c|c|c|c|c|}
\hline & \multicolumn{3}{|c|}{$\begin{array}{c}\text { Total } \\
\text { (n 1533; } 100 \cdot 0 \%) \\
\end{array}$} & \multicolumn{3}{|c|}{$\begin{array}{c}\text { Men } \\
(n 687 ; 44.2 \%) \\
\end{array}$} & \multicolumn{3}{|c|}{$\begin{array}{c}\text { Women } \\
(n 866 ; 55 \cdot 8 \%)\end{array}$} & \multirow[b]{2}{*}{$P$} \\
\hline & $n$ & Mean & SD & $n$ & Mean & SD & $n$ & Mean & SD & \\
\hline Age (years) & 1520 & $61 \cdot 6$ & $19 \cdot 3$ & 671 & $59 \cdot 0$ & $20 \cdot 5$ & 838 & $63 \cdot 7$ & $18 \cdot 0$ & $<0.001$ \\
\hline Current weight $(\mathrm{kg})$ & 1344 & 77.9 & $15 \cdot 1$ & 596 & 84.8 & $13 \cdot 8$ & 737 & $72 \cdot 3$ & 13.5 & $<0.001$ \\
\hline BMl $\left(\mathrm{kg} / \mathrm{m}^{2}\right)$ & 1304 & 29.52 & 4.97 & 585 & 29.45 & 4.40 & 709 & $29 \cdot 60$ & 5.39 & 0.877 \\
\hline Standing height $(\mathrm{cm})$ & 1337 & 162.5 & $10 \cdot 0$ & 598 & 169.9 & $7 \cdot 6$ & 728 & $156 \cdot 4$ & $7 \cdot 2$ & $<0.001$ \\
\hline WC $(\mathrm{cm})$ & 1350 & $100 \cdot 0$ & $13 \cdot 6$ & 594 & $103 \cdot 6$ & $11 \cdot 8$ & 745 & $97 \cdot 0$ & $14 \cdot 1$ & $<0.001$ \\
\hline $\mathrm{HC}(\mathrm{cm})$ & 1337 & $107 \cdot 6$ & $9 \cdot 8$ & 591 & $105 \cdot 9$ & 8.2 & 735 & 109.0 & $10 \cdot 7$ & $<0.001$ \\
\hline WHR & 1343 & 0.93 & $0 \cdot 10$ & 592 & 0.98 & 0.08 & 740 & 0.89 & 0.10 & $<0.001$ \\
\hline SBP $(\mathrm{mmHg})$ & 1259 & $138 \cdot 16$ & $19 \cdot 33$ & 552 & 140.05 & 18.44 & 697 & $136 \cdot 71$ & 19.93 & 0.0011 \\
\hline $\mathrm{DBP}(\mathrm{mmHg})$ & 1263 & 78.55 & $10 \cdot 97$ & 553 & 79.65 & $11 \cdot 28$ & 700 & 77.68 & $10 \cdot 63$ & 0.002 \\
\hline Energy intake (kJ/d) & 1445 & 10096 & 3573 & 645 & 11058 & 3741 & 800 & 9318 & 3234 & $<0.001$ \\
\hline Energy intake $(\mathrm{kcal} / \mathrm{d})$ & & 2413 & 854 & & 2643 & 894 & & 2227 & 773 & $<0.001$ \\
\hline \multicolumn{11}{|c|}{ Food consumption (servings/week) } \\
\hline Vegetables & 1444 & $27 \cdot 1$ & $14 \cdot 2$ & 644 & $26 \cdot 7$ & $14 \cdot 0$ & 800 & $27 \cdot 5$ & $14 \cdot 3$ & 0.260 \\
\hline Refined cereals & 1432 & 24.4 & $11 \cdot 0$ & 639 & $26 \cdot 0$ & 11.7 & 791 & $23 \cdot 2$ & $10 \cdot 3$ & $<0.001$ \\
\hline Fruits & 1445 & $22 \cdot 4$ & $12 \cdot 6$ & 645 & $22 \cdot 2$ & $13 \cdot 0$ & 800 & $22 \cdot 5$ & $12 \cdot 3$ & 0.737 \\
\hline Meat & 1414 & $19 \cdot 6$ & $11 \cdot 8$ & 633 & $22 \cdot 8$ & $13 \cdot 1$ & 781 & $17 \cdot 0$ & $9 \cdot 8$ & $<0.001$ \\
\hline Full-fat dairy & 1444 & $12 \cdot 2$ & $7 \cdot 1$ & 644 & $13 \cdot 0$ & 7.6 & 800 & 11.4 & $6 \cdot 7$ & $<0.001$ \\
\hline Sweets & 1431 & $10 \cdot 0$ & 7.4 & 636 & $11 \cdot 2$ & $7 \cdot 8$ & 795 & $9 \cdot 1$ & $6 \cdot 9$ & $<0.001$ \\
\hline Coffee & 1445 & $8 \cdot 1$ & 4.9 & 645 & $9 \cdot 3$ & 4.8 & 800 & $7 \cdot 1$ & 4.7 & $<0.001$ \\
\hline Fish & 1441 & $5 \cdot 1$ & 4.6 & 644 & $5 \cdot 1$ & 4.8 & 797 & $5 \cdot 2$ & 4.5 & 0.763 \\
\hline Alcoholic drinks & 1443 & $4 \cdot 2$ & 7.4 & 645 & $22 \cdot 2$ & $13 \cdot 0$ & 798 & $0 \cdot 6$ & $1 \cdot 3$ & $<0.001$ \\
\hline Potatoes & 1435 & $2 \cdot 8$ & 1.9 & 640 & 2.9 & 1.9 & 795 & $2 \cdot 8$ & 1.8 & 0.430 \\
\hline \multirow[t]{2}{*}{ Legumes } & 1437 & $2 \cdot 0$ & $1 \cdot 6$ & 642 & 1.9 & 1.6 & 795 & $2 \cdot 0$ & $1 \cdot 6$ & 0.798 \\
\hline & $n$ & \multicolumn{2}{|c|}{$\%$} & $n$ & \multicolumn{2}{|c|}{$\%$} & $n$ & \multicolumn{2}{|c|}{$\%$} & \\
\hline BMI status & 1291 & \multirow{2}{*}{\multicolumn{2}{|c|}{$17 \cdot 3$}} & 583 & \multirow{2}{*}{\multicolumn{2}{|c|}{$14 \cdot 2$}} & 708 & \multirow{2}{*}{\multicolumn{2}{|c|}{$19 \cdot 8$}} & \\
\hline Normal weight & 223 & & & 83 & & & 140 & & & 0.002 \\
\hline Overweight & 500 & \multicolumn{2}{|c|}{38.7} & 254 & \multicolumn{2}{|c|}{43.6} & 246 & \multicolumn{2}{|c|}{34.7} & \\
\hline Obese & 568 & \multicolumn{2}{|c|}{$44 \cdot 0$} & 246 & \multicolumn{2}{|c|}{$42 \cdot 2$} & 322 & \multicolumn{2}{|c|}{45.5} & \\
\hline
\end{tabular}

WC, waist circumference; HC, hip circumference; WHR, waist-to-hip ratio; SBP, systolic blood pressure; DBP, diastolic blood pressure.

$P$ values $<0.05$ are indicated in bold font; Student's $t$ test for continuous variables, $X^{2}$ test for categorical variables. 
Table 2 Disease prevalence in the study population by sex: adults from isolated Mylopotamos villages, Crete, Greece, the Hellenic Isolated Cohorts MANOLIS study

\begin{tabular}{|c|c|c|c|c|c|c|c|}
\hline \multirow[b]{2}{*}{ Disease } & \multicolumn{2}{|c|}{ Total (n 1352) } & \multicolumn{2}{|c|}{ Men $(n 566)$} & \multicolumn{2}{|c|}{ Women $(n 786)$} & \multirow[b]{2}{*}{$P$} \\
\hline & $n$ & $\%$ & $n$ & $\%$ & $n$ & $\%$ & \\
\hline Obesity & 568 & $42 \cdot 0$ & 246 & 43.5 & 322 & $41 \cdot 0$ & 0.23 \\
\hline Hypertension & 669 & 49.5 & 256 & $45 \cdot 2$ & 412 & 52.4 & $<0.001$ \\
\hline Hyperlipidaemia & 509 & 37.7 & 171 & $30 \cdot 2$ & 338) & $43 \cdot 0$ & $<0.001$ \\
\hline Arthritis & 448 & 33.1 & 119 & 21.0 & 329 & 41.9 & $<0.001$ \\
\hline Presbyopia & 415 & $30 \cdot 7$ & 154 & $27 \cdot 2$ & 261 & 33.2 & 0.0034 \\
\hline Osteoporosis & 275 & $20 \cdot 3$ & 7 & 1.2 & 268 & $34 \cdot 1$ & $<0.001$ \\
\hline Type 2 diabetes & 166 & $12 \cdot 3$ & 67 & $11 . \overline{8}$ & 99 & $12 \cdot 6$ & 0.28 \\
\hline Weak hearing & 203 & $15 \cdot 0$ & 90 & $15 \cdot 9$ & 113 & 14.4 & 0.69 \\
\hline Cataracts & 199 & 14.7 & 74 & 13.1 & 125 & $15 \cdot 9$ & 0.12 \\
\hline Chronic stress/depression & 195 & 14.4 & 31 & $5 \cdot 5$ & 164 & $20 \cdot 9$ & $<0.001$ \\
\hline Allergy & 182 & 13.5 & 60 & $10 \cdot 6$ & 122 & $15 \cdot 5$ & 0.015 \\
\hline
\end{tabular}

$P$ values $<0.05$ are indicated in bold font; $x^{2}$ test.

Table 3 Sociodemographic and lifestyle characteristics of the study population by sex: adults from isolated Mylopotamos villages, Crete, Greece, the Hellenic Isolated Cohorts MANOLIS study

\begin{tabular}{|c|c|c|c|c|c|c|c|}
\hline & \multicolumn{2}{|c|}{ Total } & \multicolumn{2}{|c|}{ Men } & \multicolumn{2}{|c|}{ Women } & \multirow[b]{2}{*}{$P$} \\
\hline & $n$ & $\%$ & $n$ & $\%$ & $n$ & $\%$ & \\
\hline Marital status & 1433 & $100 \cdot 0$ & 637 & 44.5 & 796 & 55.5 & \\
\hline Married & 1011 & $70 \cdot 6$ & 503 & 79.0 & 508 & 63.8 & $<0.001$ \\
\hline In relationship & 17 & 1.2 & 13 & $2 \cdot 0$ & 4 & 0.5 & \\
\hline Separated/divorced & 9 & $0 . \overline{6}$ & 6 & 1.0 & 3 & 0.4 & \\
\hline Single & 153 & $10 \cdot 7$ & 93 & 14.6 & 60 & 7.5 & \\
\hline Widowed & 243 & $17 \cdot 0$ & 22 & 3.5 & 221 & $27 \cdot 8$ & \\
\hline Educational attainment & 1443 & $100 \cdot 0$ & 645 & 44.7 & 798 & $55 \cdot 3$ & \\
\hline No education & 153 & $10 \cdot 6$ & 30 & 4.7 & 123 & $15 \cdot 4$ & $<0.001$ \\
\hline Primary & 909 & 63 & 416 & 64.5 & 493 & $61 \cdot 8$ & \\
\hline Secondary & 314 & 21.8 & 165 & $25 \cdot 6$ & 149 & $18 \cdot 7$ & \\
\hline Tertiary & 57 & 4.0 & 28 & 4.3 & 29 & 3.6 & \\
\hline Other & 10 & 0.7 & 6 & 0.9 & 4 & 0.5 & \\
\hline Smoking habits & 1440 & $100 \cdot 0$ & 644 & $44 \cdot 7$ & 796 & $55 \cdot 3$ & \\
\hline Current smokers & 314 & $21 \cdot 8$ & 249 & 38.7 & 65 & 8.2 & $<0.001$ \\
\hline Former smokers & 259 & $18 \cdot 0$ & 235 & $36 \cdot 5$ & 24 & 3.0 & \\
\hline \multirow[t]{2}{*}{ Never smokers } & 867 & $60 \cdot 2$ & 160 & $24 \cdot 8$ & 707 & 88.8 & \\
\hline & $n$ & Mean or SD & $n$ & Mean or SD & $n$ & Mean or SD & \\
\hline $\begin{array}{l}\text { Smoking starting age (years) } \\
\text { SD }\end{array}$ & 502 & $\begin{array}{r}18.9 \\
7.0\end{array}$ & 425 & $\begin{array}{r}18 \cdot 2 \\
6.5\end{array}$ & 72 & $\begin{array}{r}23 \cdot 1 \\
8.5\end{array}$ & $<0.001$ \\
\hline Smoking quitting age (years) & 231 & $51 \cdot 7$ & 214 & $52 \cdot 3$ & 17 & 43.5 & 0.028 \\
\hline SD & & 14.5 & & 14.2 & & $15 \cdot 7$ & \\
\hline Total smoking years & 317 & $30 \cdot 2$ & 277 & $31 \cdot 8$ & 38 & $19 \cdot 7$ & $<0.001$ \\
\hline SD & & $16 \cdot 3$ & & $16 \cdot 3$ & & $12 \cdot 7$ & \\
\hline Total years of education & 1025 & $6 \cdot 1$ & 463 & $6 \cdot 7$ & 555 & 5.5 & $<0.001$ \\
\hline SD & & 3.5 & & $3 \cdot 2$ & & $3 \cdot 7$ & \\
\hline
\end{tabular}

$P$ values $<0.05$ are indicated in bold font; $X^{2}$ test for categorical variables, Mann-Whitney test for continuous variables.

$\beta=1.015, P=0.005$ after adjusting for confounders and the rest of the dietary patterns), total cholesterol $(\beta=3.078, P=0.010 ; \beta=4.883, P=0.001$ after adjusting for confounders; $\beta=5.398, P<0.001$ after adjusting for confounders and the rest of the dietary patterns) and LDL-C $(\beta=3.977, \quad P<0.001 ; \beta=3.933, P=0.001$ after adjusting for confounders; $\beta=4.401, P=0.001$ after adjusting for confounders and the rest of the dietary patterns).

Component 4, capturing olive oil, fruit and vegetable consumption, was significantly associated with higher BMI ( $\beta=0.625, P<0.001 ; \beta=0.350, P=0.009$ after adjusting for confounders; $\beta=0.362, \quad P=0.010$ after adjusting for confounders and the rest of the dietary patterns), WHR ( $\beta=0.013, P<0.001 ; \beta=0.011, P<0.001$ after adjusting for confounders; $\beta=0.011, \quad P=<0.001$ after adjusting for confounders and the rest of the dietary patterns) and insulin levels, but not after adjustment for confounding factors for the latter association. Olive oil, fruit and vegetable consumption was found to be nominally associated with decreased SBP only after adjustment for confounders $(\beta=-1.252, \quad P$ 0.010) and taking into account both confounders and the rest of the diet $(\beta=-1 \cdot 146, P=0 \cdot 025)$. 


\section{Discussion}

In the present work we describe the collection of a population-based cohort from the isolated Mylopotamos villages on Crete, as part of the Hellenic Isolated Cohorts (HELIC) MANOLIS study (www.helic.org). The mean age of this genetically homogeneous ${ }^{(22)}$ population from mountainous Mylopotamos was 61.6 years old, denoting an elderly population.

The MANOLIS population cohort was found to be overweight to marginally obese (mean BMI $=29.5 \mathrm{~kg} / \mathrm{m}^{2}$ ). This is in keeping with other Greek ${ }^{(23,24)}$ and European ${ }^{(25)}$ elderly populations. The prevalence of obesity was $44.0 \%$ and women showed a higher tendency to be overweight than men. Generally, women were found to present higher morbidity than men, which could be attributed to the fact that the mean age for women was significantly higher. Of the population, $82.7 \%$ was overweight or obese, higher than the corresponding percentage (57\%) from the same area (Anogia) in 1990 ${ }^{(26)}$. One reason for this difference may be the study design as the previous study included only 167 elderly volunteers from Anogia, while our study assessed 1553 participants from different villages including Anogia, Zoniana, Livadia, Gonies, Axos, Astiraki, Kalivos and many others from the Mylopotamos area. Moreover, an increase in physical inactivity due to the changing modes of transportation over the years may also explain the observed shift, as the fundamental cause of obesity and overweight is the energy imbalance between energy consumed and energy expended. Hypertension affected almost half of the MANOLIS population (49.5\%), which is $20-25 \%$ lower when compared with other Greek studies in the elderly ${ }^{(23,24)}$ and another isolated population ${ }^{(27)}$, but $8 \%$ higher than the prevalence reported by a study in one of the participating villages (Anogia), 20 years ago ${ }^{(26)}$. The Greek Health Randomized Aging Study (GHRAS) is a study of 782 elderly Greeks, randomly recruited in Athens, with the aim of investigating the interactions among socio-economic, lifestyle, biochemical and psychological factors determining the health status of elderly Greeks ${ }^{(24)}$. The discrepancies for hyperlipidaemia $(37.7 \%)$ were in the same direction but of extended magnitude compared with GHRAS and the Mediterranean Islands Study (MEDIS), which found a higher prevalence of the disease (87 and $54 \%$, respectively). The prevalence of type 2 diabetes $(12.3 \%)$ in the MANOLIS population cohort is comparable with that in the general Greek population ${ }^{(28)}$ and in other isolated cohorts like the one on the island of Vis in Croatia $^{(27)}$. However, the MANOLIS population does not suffer from associated complications of the disease such as neuropathy, nephropathy, eye disease, etc. Their most prevalent diseases can be grouped into two categories: one related to CVD risk (obesity, hypertension, hyperlipidaemia, type 2 diabetes) and the other related to degenerative diseases which have age-related 
Table 5 Results from multiple linear regression analysis of the association between dietary patterns and cardiovascular risk factors among the study population ( $n$ 1183): adults from isolated Mylopotamos villages, Crete, Greece, the Hellenic Isolated Cohorts MANOLIS study

\begin{tabular}{|c|c|c|c|c|c|c|c|c|c|}
\hline & \multicolumn{3}{|c|}{ Model $1^{*}$} & \multicolumn{3}{|c|}{ Model $2 \dagger$} & \multicolumn{3}{|c|}{ Model $3 \ddagger$} \\
\hline & $\beta$ & $95 \% \mathrm{Cl}$ & $P$ & $\beta$ & $95 \% \mathrm{Cl}$ & $P$ & $\beta$ & $95 \% \mathrm{Cl}$ & $P$ \\
\hline \multicolumn{10}{|l|}{$\overline{B M l}\left(\mathrm{~kg} / \mathrm{m}^{2}\right)$} \\
\hline 'Local' diet & 0.529 & $0.272,0.786$ & $<0.001$ & 0.004 & $-0.330,0.338$ & 0.980 & 0.099 & $-0.270,0.468$ & 0.598 \\
\hline 'High fat and sugar' diet & -0.461 & $-0.718,-0.203$ & $<0.001$ & -0.289 & $-0.594,0.015$ & 0.063 & -0.193 & $-0.526,0.141$ & 0.257 \\
\hline 'Greek café/tavern' pattern & -0.016 & $-0.275,0.243$ & 0.905 & 0.241 & $-0.056,0.538$ & 0.112 & 0.202 & $-0.111,0.515$ & 0.205 \\
\hline 'Olive oil, fruits and vegetables' & 0.625 & $0.368,0.882$ & $<0.001$ & 0.350 & $0.088,0.613$ & 0.009 & 0.362 & $0.087,0.637$ & 0.010 \\
\hline \multicolumn{10}{|l|}{ WHR } \\
\hline 'Local' diet & 0.006 & $0.000,0.011$ & 0.047 & -0.005 & $-0.011,0.001$ & 0.078 & $1.04 \times 10^{-5}$ & $-0.006,0.006$ & 0.997 \\
\hline 'High fat and sugar' diet & -0.018 & $-0.024,-0.013$ & $<0.001$ & -0.004 & $-0.009,0.002$ & 0.171 & $1.4 \times 10^{-4}$ & $-0.006,0.006$ & 0.962 \\
\hline 'Greek café/tavern' pattern & 0.020 & $0.015,0.026$ & $<0.001$ & 0.012 & $0.007,0.017$ & $<0.001$ & 0.012 & $0.006,0.017$ & $<0.001$ \\
\hline 'Olive oil, fruits and vegetables' & 0.013 & $0.008,0.019$ & $<0.001$ & 0.011 & $0.007,0.016$ & $<0.001$ & 0.011 & $0.006,0.016$ & $<0.001$ \\
\hline \multicolumn{10}{|l|}{ HDL-C (mg/dl) } \\
\hline 'Local' diet & -0.247 & $-0.992,0.498$ & 0.516 & 0.918 & $-0.005,1.840$ & 0.051 & 0.670 & $-0.354,1.694$ & 0.199 \\
\hline 'High fat and sugar' diet & -0.882 & $-1.626,-0.139$ & 0.02 & -0.709 & $-1.555,0.136$ & 0.100 & -0.616 & $-1.542,0.310$ & 0.192 \\
\hline 'Greek café/tavern' pattern & -2.383 & $-3.116,-1.650$ & $<0.001$ & -0.173 & $-0.996,0.651$ & 0.681 & -0.248 & $-1 \cdot 117,0.621$ & 0.576 \\
\hline 'Olive oil, fruits and vegetables' & -0.442 & $-1.187,0.303$ & 0.245 & -0.224 & $-0.955,0.506$ & 0.547 & -0.106 & $-0.872,0.660$ & 0.786 \\
\hline \multicolumn{10}{|l|}{$\mathrm{SBP}(\mathrm{mmHg})$} \\
\hline 'Local' diet & 1.726 & $0.708,2.744$ & 0.001 & $1 \cdot 108$ & $-0.099,2.315$ & 0.072 & 0.598 & $-0.740,1.935$ & 0.381 \\
\hline 'High fat and sugar' diet & -3.946 & $-4.944,-2.948$ & $<0.001$ & -0.608 & $-1.714,0.499$ & 0.282 & -0.490 & $-1.699,0.720$ & 0.427 \\
\hline 'Greek café/tavern' pattern & -0.873 & $-1.894,0.149$ & 0.094 & 0.186 & $-0.891,1.264$ & 0.734 & 0.136 & $-0.999,1.271$ & 0.814 \\
\hline 'Olive oil, fruits and vegetables' & -0.340 & $-1.362,0.683$ & 0.515 & -1.252 & $-2.206,-0.299$ & 0.010 & $-1 \cdot 146$ & $-2.147,-0.145$ & 0.025 \\
\hline \multicolumn{10}{|l|}{ DBP $(\mathrm{mmHg})$} \\
\hline 'Local' diet & 0.612 & $0.038,1.186$ & 0.037 & 0.329 & $-0.431,1.089$ & 0.396 & 0.596 & $-0.245,1.437$ & 0.165 \\
\hline 'High fat and sugar' diet & 0.062 & $-0.513,0.637$ & 0.832 & 0.026 & $-0.671,0.722$ & 0.942 & 0.429 & $-0.332,1.190$ & 0.269 \\
\hline 'Greek café/tavern' pattern & 0.903 & $0.330,1.475$ & 0.002 & 0.846 & $0.171,1.522$ & 0.014 & 1.015 & $0.301,1.729$ & 0.005 \\
\hline 'Olive oil, fruits and vegetables' & 0.132 & $-0.443,0.707$ & 0.653 & -0.130 & $-0.731,0.472$ & 0.672 & 0.012 & $-0.681,0.641$ & 0.970 \\
\hline \multicolumn{10}{|l|}{$\mathrm{TC}(\mathrm{mg} / \mathrm{dl})$} \\
\hline 'Local' diet & $2 \cdot 067$ & $-0.27,4.405$ & 0.083 & 1.946 & $-1.161,5.053$ & 0.219 & 3.239 & $-0.191,6.669$ & 0.064 \\
\hline 'High fat and sugar' diet & $-1 \cdot 232$ & $-3.572,1.107$ & 0.302 & -1.535 & $-4 \cdot 382,1 \cdot 313$ & 0.291 & 0.673 & $-2 \cdot 429,3.776$ & 0.670 \\
\hline 'Greek café/tavern' pattern & 3.078 & $0.744,5.411$ & 0.010 & 4.883 & $2.128,7.638$ & 0.001 & $5 \cdot 398$ & $2 \cdot 488,8.308$ & $<0.001$ \\
\hline \multirow{2}{*}{\multicolumn{10}{|c|}{ LDL-C (mg/dl) }} \\
\hline & & & & & & & & & \\
\hline 'Local' diet' & 1.090 & $-0.937,3.116$ & 0.292 & 0.746 & $-1.937,3.429$ & 0.585 & $2 \cdot 111$ & $-0.853,5.074$ & 0.163 \\
\hline 'High fat and sugar' diet & 0.038 & $-1.989,2.065$ & 0.971 & -0.692 & $-3.151,1.767$ & 0.581 & 1.007 & $-1.673,3.688$ & 0.461 \\
\hline 'Greek café/tavern' pattern & 3.977 & $1.962,5.991$ & $<0.001$ & 3.933 & $1.550,6.310$ & 0.001 & 4.401 & $1.886,6.916$ & 0.001 \\
\hline 'Olive oil, fruits and vegetables' & 1.136 & $-0.891,3.162$ & 0.272 & 0.888 & $-1.233,3.009$ & 0.411 & 1.368 & $-0.848,3.585$ & 0.226 \\
\hline \multicolumn{10}{|l|}{ Glucose (mg/dl) } \\
\hline 'Local' diet & 4.861 & $3.466,6.256$ & $<0.001$ & 4.618 & $3.025,6 \cdot 212$ & $<0.001$ & 4.026 & $2 \cdot 259,5.794$ & $<0.001$ \\
\hline 'High fat and sugar' diet & -4.780 & $-6 \cdot 176,-3 \cdot 384$ & $<0.001$ & -1.660 & $-3.144,-0.176$ & 0.028 & -0.987 & $-2.595,0.621$ & 0.229 \\
\hline 'Greek café/tavern' pattern & -1.027 & $-2.448,0.394$ & 0.157 & -1.254 & $-2.691,0.183$ & 0.087 & -1.053 & $-2.553,0.448$ & 0.169 \\
\hline 'Olive oil, fruits and vegetables' & -0.208 & $-1 \cdot 631,1214$ & 0.774 & -1.303 & $-2.578,-0.027$ & 0.045 & -0.490 & $-1.813,0.833$ & 0.467 \\
\hline \multicolumn{10}{|l|}{ Insulin $(\mu \mathrm{IU} / \mathrm{ml})$} \\
\hline 'Local' diet & 0.389 & $-0.364,1.142$ & 0.311 & -0.619 & $-1.602,0.3636$ & 0.216 & -0.141 & $-1.230,0.948$ & 0.800 \\
\hline 'High fat and sugar' diet & 0.433 & $-0.321,1.186$ & 0.360 & 0.678 & $-0.226,1.582$ & 0.141 & 0.765 & $-0.226,1.756$ & 0.130 \\
\hline 'Greek café/tavern' pattern & 0.631 & $-0.121,1.384$ & $0 \cdot 100$ & 0.156 & $-0.719,1.032$ & 0.726 & 0.324 & $-0.600,1.249$ & 0.491 \\
\hline 'Olive oil, fruits and vegetables' & 0.997 & $0.245,1.748$ & 0.009 & 0.635 & $-0.142,1.411$ & 0.109 & 0.637 & $-0.178,1.452$ & 0.125 \\
\hline
\end{tabular}

$\ddagger$ Model 3: adjusted for age, sex, BMI (where applicable), medication (where applicable), physical activity level, smoking, energy intake and the remaining food patterns scores. 
pathologies (arthritis, presbyopia, osteoporosis, weak hearing, cataracts).

Meat intake of the MANOLIS population was three to eight times higher than that of the Attica general population $^{(29)}$ and other Greek elderly populations ${ }^{(23,24)}$. This is consistent with the geography of this mountainous isolated area and the tradition that has been preserved for centuries, with the local primary occupation being livestock farming. The increased alcohol consumption mainly by men reveals the social and cultural aspect of drinking. Men frequenting traditional cafés/bars drink and treat with their homemade alcoholic drink (raki) all visitors as a sign of hospitality.

The vast majority of the population was married ( $70.6 \%)$, while less than $1 \%$ were separated/divorced, demonstrating the traditional character of the population for which family is the core of social life, a characteristic that is weaker in populations living in more urban areas of Greece ${ }^{(24,30)}$. Most of the participants had attained primary education, with men more educated than women, a characteristic similar to an urban elderly population (GHRAS) but not to a younger urban Greek population ${ }^{(28)}$ which has almost double the number of total years of education. This is a characteristic typical of rural areas, where agriculture is the main occupation. Educational attainment in the MANOLIS population is further curtailed by participant age and the effect of the onset of World War II.

Men were heavy smokers while the majority of women had never smoked. The percentage of male current smokers in MANOLIS is three times that in GHRAS ( $4.7 \%$ ), while the proportion of females who are current and ex-smokers is much lower than in GHRAS $(6 v .16 \%)^{(24)}$. Again this demonstrates the traditional nature of the particular society that preserves etiquettes and standards of the past. However, smoking in men was considerably lower (38\%) compared with $57 \%$ in 1960, when a Cretan population was studied for the first time as part of the Seven Countries Study ${ }^{(31)}$.

Serum glucose levels were the same as in GHRAS ${ }^{(24)}$, while HDL-C levels in MANOLIS were found to be slightly $(7-9 \mathrm{mg} / \mathrm{dl})$ lower than those of other elderly Greek and European populations ${ }^{(24,25)}$ although the levels appear consistent with previous studies in the area over the past 30 years $^{(32-35)}$. Our population from Mylopotamos had LDL-C levels that were $25-45 \mathrm{mg} / \mathrm{dl}$ lower compared with the mean level of both studies among the elderly in Attica $^{(24)}$ and Anogia ${ }^{(32)}$. Total cholesterol levels were found to be $20-40 \mathrm{mg} / \mathrm{dl}$ lower than those of other studies from Greece and the local area ${ }^{(24,32,34,35)}$, and resembled the levels found from the Seven Countries Study ${ }^{(31)}$ in the 1960s. This highlights to some extent the Cretan paradigm of life expectancy and health.

The Mediterranean diet, one of the most studied dietary patterns, has been linked to increased survival, reduced risk of cancers and CVD and increased longevity ${ }^{(36-38)}$, and has historically been derived from Crete, the origin of our study population. However, taking into account the geographical diversity of this island that affects the availability of food and living conditions, the dietary and lifestyle habits of a mountainous population may differ substantially from those of a valley population. In order to characterize the unique diet of this particular mountainous population from Mylopotamos, PCA, an a posteriori technique, was used to investigate patterns in the dietary data. A posteriori patterns are generated by empirically obtained data at hand ${ }^{(39)}$ and thus they do not necessarily identify 'healthy' patterns, but patterns that depict the commonly recognized eating habits of the particular population. The extracted components are based on subjective decisions. The derived patterns found were: the 'local' diet pattern, which represents a traditional diet of the native population; the 'high fat and sugar' diet pattern, which in addition to high sweet and fat consumption includes the preference for processed and pre-packed food; the 'Greek café/tavern' pattern that is characterized by coffee and alcohol consumption, fries and olives as side dishes for alcohol; and the 'olive oil, fruits and vegetables' pattern with olive oil, fresh fruit and vegetable consumption.

The associations of these patterns with several cardiovascular risk factors like BMI, WHR, total cholesterol, HDL-C, LDL-C, SBP, DBP, glucose and insulin were evaluated, after removing energy under-reporters. Under-reporting is more frequent among overweight individuals ${ }^{(40)}$, like our study population.

Data analysis revealed no association of any of the derived dietary patterns with HDL-C and insulin. Others like Fung et $a l^{(41)}$ observed a negative association between insulin and the prudent dietary pattern that included fruits, vegetables, poultry, fish, whole grains and legumes, and a positive association with the Western pattern diet characterized by higher consumption of red and processed meat, French fries, eggs, high-fat dairy products, sweets and refined grains.

The olive oil, fruits and vegetables diet pattern was negatively associated with SBP, suggesting that the antioxidant compounds ${ }^{(42)}$ of this specific pattern might play a protective role. Olive oil has a dominant presence in the Mediterranean diet, which has been inversely related to SBP and $\mathrm{DBP}^{(43,44)}$. Similar findings have been published before ${ }^{(45,46)}$. However, the olive oil, fruits and vegetables diet pattern was also found to be associated with high BMI and WHR, an effect that could be attributed to the oil, which is the most energy-dense component of the pattern. When the compliance to this pattern (scores) was divided into tertiles, it was the medium tertile, not the higher tertile, that was significantly associated with increased risk for overweight/obesity. The latter might be due to the lack of discriminating capacity of this pattern considering the broadly high consumption of olive oil, fruits and vegetables in the area. In addition, it can be 
attributed to the fact that the majority of the specific population is overweight or obese, as well as to the previously mentioned protective role against other cardiometabolic risk factors such as blood pressure. In the Di@bet.es study $^{(47)}$, a negative association was found between the prevalence of metabolic complications and olive oil intake, which explains the so-called 'Spanish paradox' where the morbidity and mortality rates for CVD are lower than expected from the high prevalence of metabolic complications, whether or not related to obesity ${ }^{(48)}$

The local diet pattern was associated only with higher glucose levels, in line with other studies where dietary patterns characterized by a higher intake of animal fat, mainly red meat and meat products, saturated fat, cholesterol and full-fat dairy products have been associated with increased CVD risk and death rate ${ }^{(29,49)}$. This pattern was not found to be significantly associated with any other cardiometabolic risk factors, which seems surprising considering the unhealthy nature of the diet. This could be explained by the counterpoising effect of low carbohydrate and high fat content in increasing HDL-C levels ${ }^{(50)}$, an independent cardioprotective factor. Furthermore, the absence from the local pattern of any pre-packed or processed products reduces the load of trans-fatty acids. These compounds have been shown to have well-established adverse cardiovascular effects ${ }^{(51)}$ and decrease HDL-C ${ }^{(52)}$. In addition, the HELIC-MANOLIS population was found to have a low-frequency cardioprotective genetic variant associated with increased HDL-C levels, at a frequency forty times higher than the general Greek population ${ }^{(53)}$. This shepherd population used to spend most of their time high in the mountains with their herds, so goat meat and milk/cheese were in abundance and in fact the only foods available to them to eat at times.

The Greek café/tavern pattern, a unique and very characteristic pattern for the culture of this population, was found to be associated with both BMI and WHR, demonstrating the relationship of this pattern with adiposity, as well as total cholesterol and DBP, indices that contribute more to the cardiometabolic risk. This finding is in absolute accordance with the established knowledge about cardiovascular risk factors ${ }^{(1)}$ taking into account the alcohol consumption as a component of this pattern, being in the same direction as observed in the ATTICA study where a diet pattern indicated by alcohol consumption was associated with CVD risk $^{(29)}$.

Our study had an observational, cross-sectional design and therefore shares the limitations of this approach. It is not appropriate to draw causal effect implications or to generalize based on the results from this particular Mylopotamos population. Residual confounding may also exist because of unused/unmeasured variables. Another limitation of the study is the self-reported medical history and medication data. In addition, the FFQ was not validated in this specific population, although it has been validated in the general Greek population ${ }^{(15)}$. The subjective nature of the decision making in components extraction is a limitation of PCA application. Lastly, we have not corrected our results for the number of tests carried out. We have tested nine outcomes of interest and hence the stringent Bonferroni-corrected $P$ value is 0.0056 $(=0 \cdot 05 / 9)$. Using this significance threshold, the associations of the local dietary pattern with glucose levels, the Greek café/tavern pattern with WHR and cholesterol levels, and the olive oil, fruits and vegetables diet pattern with WHR, remain significant. The associations of the Greek café/tavern pattern with DBP and LDL-C levels, and of the olive oil, fruits and vegetables diet pattern with BMI and SBP, become suggestive as they no longer reach nominal significance.

\section{Conclusion}

The Cretan MANOLIS population is an isolated, elderly population with hypertension, obesity and hyperlipidaemia as the most prevalent diseases, the percentages of which resemble those of the general population. It can be described as a traditional society with family at the core of social life. The very characteristic dietary patterns, local and Greek café/tavern, identified in our study, are associated with overweight/obesity and other cardiovascular risk factors such as raised glucose, blood pressure and cholesterol levels, predicating their characterization as unhealthy. Consequently, the guidelines for decreasing cardiovascular risk should be applied to this population, through providing further information, raising awareness and policy making in these isolated areas. The burden of dietary compounds like high fat and red meat consumption in this population is not depicted in the intermediate cardiovascular manifestations to the expected extent, indicating compensatory procedures that require further investigation through gene-environment interaction analyses.

\section{Acknowledgements}

Acknowledgements: The authors thank the residents of the Mylopotamos villages for taking part. The MANOLIS study is dedicated to the memory of Manolis Giannakakis, 1978-2010. The MANOLIS study has been supported by many individuals who have contributed to sample collection (including Olina Balafouti, Christina Batzaki, Georgios Daskalakis, Eleni Emmanouil, Chrisoula Giannakaki, Margarita Giannakopoulou, Anastasia Kaparou, Vasiliki Kariakli, Stella Koinaki, Dimitra Kokori, Maria Konidari, Hara Koundouraki, Dimitris Koutoukidis, Eirini Mamalaki, Eirini Mpamiaki, George Pananikolaou, Maria Tsoukana, Dimitra Tzakou, Katerina Vosdogianni and Niovi Xenaki), data entry (Eleni Zengini, Thanos Antonos and Dimitra Papagrigoriou) and research 
administration (Anja Kolb-Kokocinski, Carol Smee and Danielle Walker). Financial support: This work was funded by the Wellcome Trust (grant number 098051) and the European Research Council (grant number ERC-2011StG 280559-SEPI). The Wellcome Trust had no role in the design, analysis or writing of this article. The European Research Council had no role in the design, analysis or writing of this article. This work has taken place under the auspices of the Alexander S. Onassis Public Benefit Foundation Scholarship Program for Hellenes (grant number GZI 049/2012-2013). The Alexander S. Onassis Public Benefit Foundation had no role in the design, analysis or writing of this article. Conflict of interest: None. Authorship: E.Z. and G.D. contributed equally to this work. G.D. formulated the research question(s). E.Z. and G.D designed the study. A.-E.F., P.S., V.K., C.K. and E.T. carried out the study. A.-E.F., N.W.R., A.M. and A.G. analysed the data. A.-E.F. wrote the article. Ethics of buman subject participation: This study was conducted according to the guidelines laid down in the Declaration of Helsinki and all procedures involving human subjects were approved by the Bioethics Committee of Harokopio University of Athens. Written informed consent was obtained from all participants.

\section{Supplementary material}

To view supplementary material for this article, please visit https://doi.org/10.1017/S1368980016003207

\section{References}

1. World Health Organization (2015) Cardiovascular Diseases (CVDs). Fact Sheet no. 317. Geneva: WHO.

2. Zimmet P, Alberti KG \& Shaw J (2001) Global and societal implications of the diabetes epidemic. Nature $\mathbf{4 1 4}$ 782-787.

3. Palacios C, Perez CM, Guzman M et al. (2011) Association between adiposity indices and cardiometabolic risk factors among adults living in Puerto Rico. Public Health Nutr 14, 1714-1723.

4. Ashwell M, Gunn P \& Gibson S (2012) Waist-to-height ratio is a better screening tool than waist circumference and BMI for adult cardiometabolic risk factors: systematic review and meta-analysis. Obes Rev 13, 275-286.

5. Kahn R, Buse J, Ferrannini E et al. (2005) The metabolic syndrome: time for a critical appraisal: joint statement from the American Diabetes Association and the European Association for the Study of Diabetes. Diabetes Care $\mathbf{2 8}$, 2289-2304.

6. Grundy SM (2007) Metabolic syndrome: a multiplex cardiovascular risk factor. J Clin Endocrinol Metab 92, 399-404.

7. Yamaoka K \& Tango T (2012) Effects of lifestyle modification on metabolic syndrome: a systematic review and meta-analysis. BMC Med 10, 138.

8. Visscher PM, Brown MA, McCarthy MI et al. (2012) Five years of GWAS discovery. Am J Hum Genet 90, 7-24.

9. Speliotes EK, Willer CJ, Berndt SI et al. (2010) Association analyses of 249,796 individuals reveal 18 new loci associated with body mass index. Nat Genet 42, 937-948.
10. National Research Council, National Committee on Diet and Health (1989) Diet and Health: Implications for Reducing Chronic Disease Risk. Washington, DC: National Academies Press.

11. Keys A, Menotti A, Karvonen MJ et al. (1986) The diet and 15-year death rate in the seven countries study. Am J Epidemiol 124, 903-915.

12. Friedewald WT, Levy RI \& Fredrickson DS (1972) Estimation of the concentration of low-density lipoprotein cholesterol in plasma, without use of the preparative ultracentrifuge. Clin Chem 18, 499-502.

13. Yannakoulia M, Melistas L, Solomou E et al. (2007) Association of eating frequency with body fatness in preand postmenopausal women. Obesity (Silver Spring) 15, 100-106.

14. Ainsworth BE, Haskell WL, Herrmann SD et al. (2011) 2011 Compendium of Physical Activities: a second update of codes and MET values. Med Sci Sports Exerc 43, $1575-1581$.

15. Bountziouka V, Bathrellou E, Giotopoulou A et al. (2012) Development, repeatability and validity regarding energy and macronutrient intake of a semi-quantitative food frequency questionnaire: methodological considerations. Nutr Metab Cardiovasc Dis 22, 659-667.

16. Ministry of Health and Welfare, Supreme Scientific Health Council (1999) Dietary guidelines for adults in Greece. Arch Hellenic Med 16, 516-524.

17. Goldberg GR, Black AE, Jebb SA et al. (1991) Critical evaluation of energy intake data using fundamental principles of energy physiology: 1. Derivation of cut-off limits to identify under-recording. Eur J Clin Nutr 45, 569-581.

18. World Health Organization (1985) Energy and Protein Requirements. Report of a Joint FAO/WHO/UNU Expert Consultation. WHO Technical Report Series no. 724. Geneva: WHO.

19. Mardia KV, Kent JT \& Bibby JM (1979) Multivariate Analysis. New York: Academic Press.

20. Hu FB (2002) Dietary pattern analysis: a new direction in nutritional epidemiology. Curr Opin Lipidol 13, 3-9.

21. Expert Panel on Detection, Evaluation, and Treatment of High Blood Cholesterol In Adults (2001) Executive Summary of The Third Report of The National Cholesterol Education Program (NCEP) Expert Panel on Detection, Evaluation, And Treatment of High Blood Cholesterol In Adults (Adult Treatment Panel III). JAMA 285, 2486-2497.

22. Panoutsopoulou K, Hatzikotoulas K, Xifara DK et al. (2014) Genetic characterization of Greek population isolates reveals strong genetic drift at missense and trait-associated variants. Nat Commun 5, 5345.

23. Tyrovolas S, Zeimbekis A, Bountziouka V et al. (2009) Factors associated with the prevalence of diabetes mellitus among elderly men and women living in Mediterranean Islands: the MEDIS Study. Rev Diabet Stud 6, 54-63.

24. Kanoni S \& Dedoussis GV (2008) Design and descriptive characteristics of the GHRAS: the Greek Health Randomized Aging Study. Med Sci Monit 14, CR204-CR212.

25. Dedoussis GV, Kanoni S, Mariani E et al. (2008) Mediterranean diet and plasma concentration of inflammatory markers in old and very old subjects in the ZINCAGE population study. Clin Chem Lab Med 46, 990-996.

26. Kafatos A, Diacatou A, Labadarios D et al. (1993) Nutrition status of the elderly in Anogia, Crete, Greece. J Am Coll Nutr 12, 685-692.

27. Missoni S (2009) Metabolic syndrome among the inhabitants of the island of Vis. Coll Antropol 33, 1281-1287.

28. Athyros VG, Bouloukos VI, Pehlivanidis AN et al. (2005) The prevalence of the metabolic syndrome in Greece: the MetS-Greece Multicentre Study. Diabetes Obes Metab 7, 397-405. 
29. Panagiotakos D, Pitsavos C, Chrysohoou C et al. (2009) Dietary patterns and 5-year incidence of cardiovascular disease: a multivariate analysis of the ATTICA study. Nutr Metab Cardiovasc Dis 19, 253-263.

30. Panagiotakos DB, Zeimbekis A, Boutziouka V et al. (2007) Long-term fish intake is associated with better lipid profile, arterial blood pressure, and blood glucose levels in elderly people from Mediterranean islands (MEDIS epidemiological study). Med Sci Monit 13, CR307-CR312.

31. Moschandreas J, Kafatos A, Aravanis C et al. (2005) Long-term predictors of survival for the Seven Countries Study cohort from Crete: from 1960 to 2000. Int J Cardiol 100, 85-91.

32. Manios Y, Antonopoulou S, Kaliora AC et al. (2005) Dietary intake and biochemical risk factors for cardiovascular disease in two rural regions of Crete. J Physiol Pharmacol 56, Suppl. 1, 171-181.

33. Aravanis C, Mensink RP, Corcondilas A et al. (1988) Risk factors for coronary heart disease in middle-aged men in Crete in 1982. Int J Epidemiol 17, 779-783.

34. Kafatos A, Schlienger JL, Deslypere JP et al. (1991) Nutritional status: serum lipids. Euronut SENECA investigators. Eur J Clin Nutr 45, Suppl. 3, 53-61.

35. Sandker GW, Kromhout D, Aravanis C et al. (1993) Serum cholesteryl ester fatty acids and their relation with serum lipids in elderly men in Crete and The Netherlands. Eur J Clin Nutr 47, 201-208.

36. Sofi F, Abbate R, Gensini GF et al. (2010) Accruing evidence on benefits of adherence to the Mediterranean diet on health: an updated systematic review and meta-analysis. Am J Clin Nutr 92, 1189-1196.

37. Trichopoulou A, Costacou T, Bamia C et al. (2003) Adherence to a Mediterranean diet and survival in a Greek population. N Engl J Med 348, 2599-2608.

38. Knoops KT, de Groot LC, Kromhout D et al. (2004) Mediterranean diet, lifestyle factors, and 10-year mortality in elderly European men and women: the HALE project. JAMA 292, 1433-1439.

39. Trichopoulos D \& Lagiou P (2001) Dietary patterns and mortality. Br J Nutr $\mathbf{8 5}, 133-134$.

40. McCrory MA, Howarth NC, Roberts SB et al. (2011) Eating frequency and energy regulation in free-living adults consuming self-selected diets. J Nutr 141, 148-153.

41. Fung TT, Rimm EB, Spiegelman D et al. (2001) Association between dietary patterns and plasma biomarkers of obesity and cardiovascular disease risk. Am J Clin Nutr 73, 61-67.
42. Su Q, Rowley KG, Itsiopoulos C et al. (2002) Identification and quantitation of major carotenoids in selected components of the Mediterranean diet: green leafy vegetables, figs and olive oil. Eur J Clin Nutr 56, 1149-1154.

43. Psaltopoulou T, Naska A, Orfanos P et al. (2004) Olive oil, the Mediterranean diet, and arterial blood pressure: the Greek European Prospective Investigation into Cancer and Nutrition (EPIC) study. Am J Clin Nutr 80, 1012-1018.

44. Papamichael CM, Karatzi KN, Papaioannou TG et al. (2008) Acute combined effects of olive oil and wine on pressure wave reflections: another beneficial influence of the Mediterranean diet antioxidants? J Hypertens 26, 223-229.

45. Alonso A, Ruiz-Gutierrez V \& Martinez-Gonzalez MA (2006) Monounsaturated fatty acids, olive oil and blood pressure: epidemiological, clinical and experimental evidence. Public Health Nutr 9, 251-257.

46. Ferrara LA, Raimondi AS, d'Episcopo L et al. (2000) Olive oil and reduced need for antihypertensive medications. Arch Intern Med 160, 837-842.

47. Gutierrez-Repiso C, Soriguer F, Rojo-Martinez G et al. (2014) Variable patterns of obesity and cardiometabolic phenotypes and their association with lifestyle factors in the Di@bet.es study. Nutr Metab Cardiovasc Dis 24, 947-955.

48. Serra-Majem L, Ribas L, Tresserras R et al. (1995) How could changes in diet explain changes in coronary heart disease mortality in Spain? The Spanish paradox. Am J Clin Nutr $\mathbf{6 1}$, 6 Suppl., 1351S-1359S.

49. Walker P, Rhubart-Berg P, McKenzie S et al. (2005) Public health implications of meat production and consumption. Public Health Nutr 8, 348-356.

50. Katan MB (1998) Effect of low-fat diets on plasma highdensity lipoprotein concentrations. Am J Clin Nutr 67, 3 Suppl., 573S-576S.

51. Michas G, Micha R \& Zampelas A (2014) Dietary fats and cardiovascular disease: putting together the pieces of a complicated puzzle. Atherosclerosis 234, 320-328.

52. Mozaffarian D, Katan MB, Ascherio A et al. (2006) Trans fatty acids and cardiovascular disease. $N$ Engl J Med 354, 1601-1613.

53. Tachmazidou I, Dedoussis G, Southam L et al. (2013) A rare functional cardioprotective APOC3 variant has risen in frequency in distinct population isolates. Nat Commun 4, 2872 . 